\title{
Fully Automatic Detection and Segmentation Approach for Juxta- Pleural Nodules From CT Images
}

\author{
Vijayalaxmi Mekali, KSIT, Bengaluru, India \\ Girijamma H. A., RNSIT, Bengaluru, India
}

\begin{abstract}
Early detection of all types of lung nodules with different characters in medical modality images using computer-aided detection is the best acceptable remedy to save the lives of lung cancer sufferers. But accuracy of different types of nodule detection rates is based on chosen segmented procedures for parenchyma and nodules. Separation of pleural from juxta-pleural nodules (JPNs) is difficult as intensity of pleural and attached nodule is similar. This research paper proposes a fully automated method to detect and segment JPNs. In the proposed method, lung parenchyma is segmented using iterative thresholding algorithm. To improve the nodules detection rate separation of connected lung lobes, an algorithm is proposed to separate connected left and right lung lobes. The new method segments JPNs based on lung boundary pixels extraction, concave points extraction, and separation of attached pleural from nodule. Validation of the proposed method was performed on LIDC-CT images. The experimental result confirms that the developed method segments the JPNs with less computational time and high accuracy.
\end{abstract}

\section{KEYWORDS}

Benign and Malignant Tumors, Computed Tomography, Computer-Aided Detection, Connected Lung Lobes, Juxta-Vascular Nodules, Lung Boundary Pixels, Lung Nodules, Lung Parenchyma Segmentation

\section{INTRODUCTION}

Lung cancer is disease with highest death rate as compared to breast, prostate, brain and cervical cancers. Even with number of available detection methodologies for lung cancer, life span of patient with stage III and IV lung cancer is still not improving as expected. Thus early stage lung nodules detection followed by the proper treatment is best choice to avoid conversion of early stage nodules into malignant tumors and to reduce mortality rate. Lung nodules possible size ranges from $3 \mathrm{~mm}$ to $30 \mathrm{~mm}$. Small, non-cancerous and smooth boundary lung nodules are benign nodules. Large, cancerous and irregular boundary lung tumors are knows as malignant nodules. Non-detected benign nodules may get converts into cancer tumors. Based on nodule's intensity variation, texture and additional connected components which are not a part of nodules, lung nodules are differentiated into well circumscribed, Juxta-Vascular Nodules (JVN) - attached to blood vessels, Juxta-Pleural Nodules (JPN) - attached to lung pleural and Ground Glass Opacity (GGO) nodules as in Figure 1. Further nodules at next level of classification on their solidity feature are solid, partly solid or non-solid nodules as in Figure 2. 
Medical imaging modalities like X-ray, Magnetic Resonance Imaging (MRI), Diffusion Weight MRI (DW-MRI), Computed Tomography (CT), Ultrasound (US) and other modalities have been part of medical routine for lung cancer detection. On medical images lung nodules appears as white patch. CT is GOLD STANDARD modality for detection of all types and stages lung nodules. Figure 3 shows the pixels value of all possible parts of lung CT image. As CT generates huge amount of images in single scan, interpretation of these images by radiologist to study the characteristic of nodules for further treatment is time consuming. Core of medical procedures to confirm the presence and severity of lung cancer are Computer Aided Detection (CAD) systems. These systems provides useful and accurate information about nodules for a radiologist to draw the useful conclusion about lung nodules (characteristic, size, benign or malignant), thus improves treatment option. With a use of available technologies and to make quick diagnosis of cancer, CAD systems can be implemented to provide remote E-health care services (Ashutosh Sharma \& Rajiv Kumar 2019).

Figure 1. a) Well-Circumscribed Nodule b) Juxta-Vascular Nodule c) Juxta-Pleural Nodule
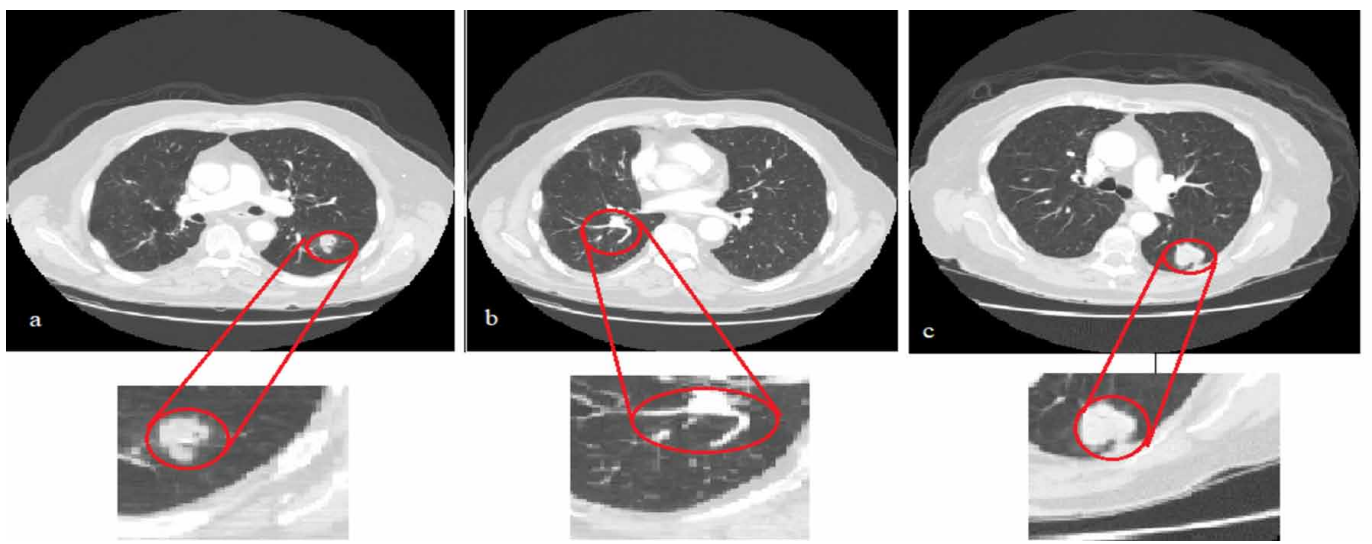

Figure 2. a) Solid nodule b) Part-solid nodule c) Non-solid nodule
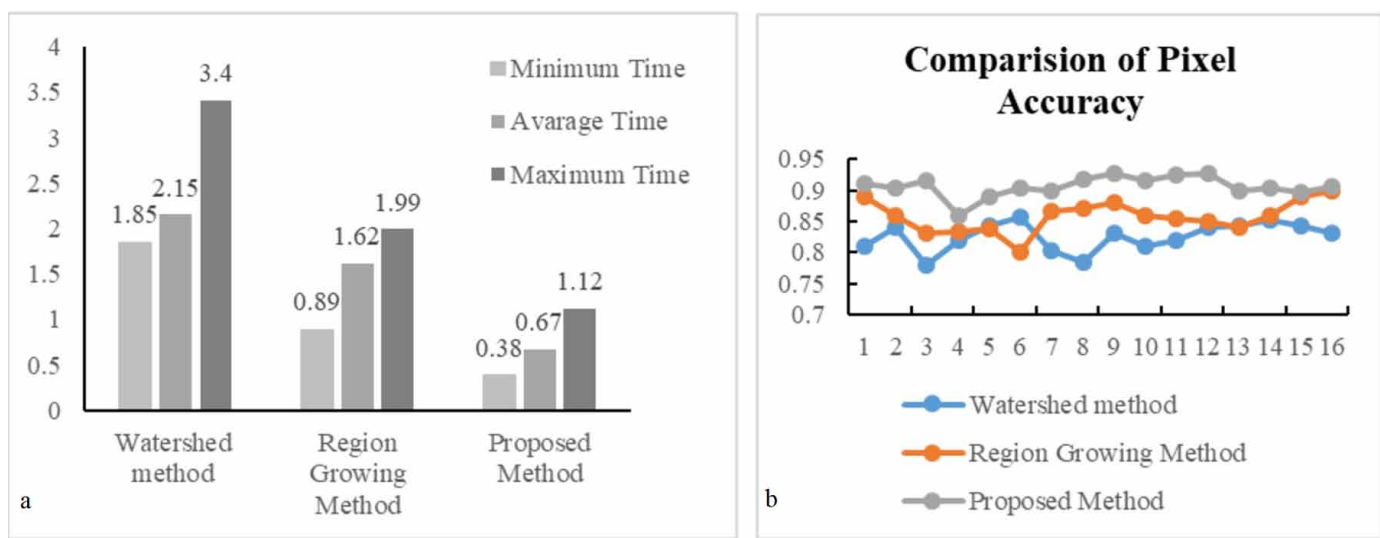
Figure 3. Histogram of original CT image that shows the pixel values of fat, bones, lung parenchyma and background.
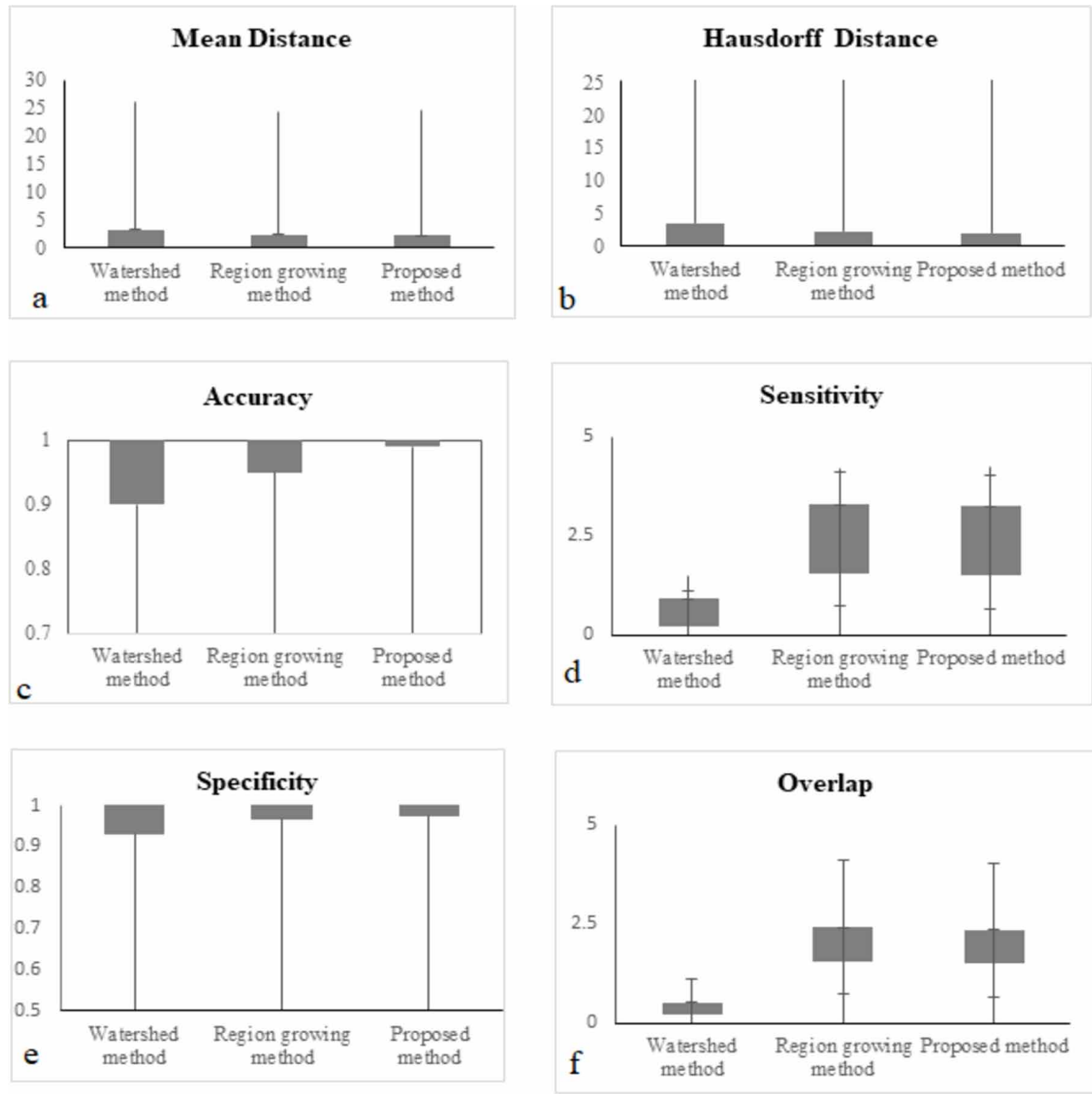

\section{LITERATURE REVIEW}

(K. Suzuki \& K. Doi, 2004; K. Suzuki \& K. Doi, 2005; Suzuki, Z. H. Shi, \& J. Zhang, 2008) explained implementation of new CAD system based on supervised filter - Massive Training Artificial Neural Network (MTANN) to detect and classify lung nodules. With this new approach $97 \%$ sensitivity was achieved with reduced false positive rate.

(S. Diciotti, G. Picozzi, M. Falchini, M. Mascalchi, N. Villari, \& G. Valli, 2008) proposed novel segmentation technique based on significant properties such as expert's knowledge, geodic distance computation and user interaction to segment nodules with lesser diameter (phantom 5.3-11 and vivo 5-9.8). This method also considered JVN and JPN segmentation. CT images with 157 nodules were taken from Lung Image Database Consortium (LIDC) public database and achieved sensitivity of $88.5 \%$.

(Q. Wang, E. Song, R. Jin, P. Han, X. Wang, Y. Zhou, \& J. Zeng, 2009) explained lung nodules segmentation algorithm based on information present in adjacent $\mathrm{CT}$ slices and multidirectional 
relationship to segment different types of nodules. Data set 1 and data set 2 of CT images from LIDC were used for validation of presented methodology and 75\% accuracy was achieved.

(T. Messay., Hardie R. C., \& Rogers S. K, 2010) proposed computationally efficient lung nodules detection method. In this method after lung boundary extraction, threshold and morphological operations were used to segment nodules. Features set of totally 245 features from each segmented nodule was considered and finally optimal feature set was determined. Two classifier FLD classifier and Quadratic classifier were used for nodules classification. Since FLD performance was outstanding it was considered for implementation of new CAD system. Different image processing techniques has been playing a major role in medical fields to diagnose different types of diseases. Among that cancer detection CAD systems have been developed by many researcher using different image processing techniques.

(Qi, C. 2010; Tong, J., 2008) proposed lung parenchyma segmentation methods based on different thresholding techniques to detect pulmonary nodules from CT images. (Li, J., Zheng, B., Liang, H. \& Deng, Y. L., 2013) developed new parenchyma method using RG and morphological operations. 2D convexhull algorithm was used to repair the extracted lung boundary. (Geng, H., Bian, Z., Yang, J., Tan, W., \& Zhao, D., 2014) presented lung parenchyma extraction by iterative thresholding and RG based on automatic seed selection. (Gong, J., Wang, L. \& Wang, Y., 2015) used thresholding and FCM clustering techniques for lung parenchyma segmentation to detect the lung nodules. (Ashutosh Sharma, Mohd Dilshad Ansari \& Rajiv Kumar., 2018) discussed different edge detection techniques in detection and segmentation of different types of objects in images, anatomical structures in medical image.

(T. Kubota, A. K. Jerebko, M. Dewan, M. Salganicoff, \& A. Krishnan, 2011) proposed three stage lung nodules segmentation method. Segmentation of Solid, part-solid, non-solid nodules of type JPN and JVN were considered. In First stage lung parenchyma and denser structures segmentation was performed, in second stage JPN core location was achieved by foreground's Euclidian Distance Transformation (EDT) and finally separation of JPN was done with Region Growing (RG) method. The work was tested on two data sets of CT images of LIDC.

(Farag, H. E. A. El Munim, J. H. Graham, \& A. a Farag, 2013) developed lung lesions shape model based on level set technique and image intensity to segment lung nodules from CT images. This new model was not dependent of nodules type and location. CT images with total of 742 nodules from four different databases were used in evaluation of new model.

(D. M. Campos, A. Simoes, I. Ramos, \& A. Campilho, 2014) proposed two stage supervised lung nodules segmentation method. In this model nodules segmentation was achieved with three independent methods: RG with volumetric index, RG with convergence index filter, and K- Nearest Neighbor (KNN) classifier. Features such as shape, intensity and contrast of segmented lung nodules were used to train Artificial Neural for final nodules segmentation.

(Mansoor, U. Bagci, Z. Xu, B. Foster, K. N. Olivier, J. M. Elinoff, et al, 2014) proposed new lung parenchyma segmentation procedure to detect nodules. In this method segmented lung parenchyma by fuzzy connectedness algorithm was compared with rib cage information based lung volume. Presence of pathology was confirmed with difference in both lung volumes. And texture features were used detect pathologies such as nodules, Ground glass, consolidations and small nodules also.

(Xuanping Li, Xue Wang, Yixiang Dai, \& Pengbo Zhang, 2015) has presented recursive strategy for extracting lung tissue from lung CT image using geometric active contour method. Segmentation of volumetric CT slices was carried out, in which the segmentation result of previous slice was used to segment the next slice to compensate the minimal changes between the adjacent slices. Left-right lung lobes fusion and segmentation of JPN was also considered in the proposed procedure. (Yong qiang Tan, 2013) used watershed, active contours, and Markov random field for detection of lung nodules in thoracic CT images. (Ezhil, 2016) developed lung nodules segmentation method based on active contour model and FCM clustering. 
(Acharjya, D. P., \& Chowdhary, C. L. 2018; C. L. Chowdhary \& D. Acharjya, 2018; Chowdhary, C. L., \& Acharjya, D. P. 2016; Chowdhary, C. L., \& Acharjya, D. P. 2016) proposed different hybridization techniques in their work for breast cancer detection from mammogram images. Algorithms used in their work were Intuitionistic fuzzy set, intuitionistic fuzzy histogram hyperbolization, Possibilistic Fuzzy C-Mean (PFCM) clustering algorithm and intuitionistic Fuzzy C-Mean algorithm. (Chowdhary, C. L., Sai, G. V. K., \& Acharjya, D. P. 2016) presented new method to reduce false assumption in breast cancer detection. In this approach extra slices were eliminated in pre-processing step, homogeneous block method was applied to segment the image and finally color quantization was used. (Chowdhary, C. L., \& Acharjya, D. P. 2017) developed new hybrid segmentation method for medical images segmentation based on PFCM and Exponential Fuzzy C-Means algorithms (EFCM). Segmentation accuracy of $97.4 \%$ was achieved.

(Heewon Chung, Hoon Ko, Se Jeong Jeon, Kwon-Ha Yoon., \& Jinseok Lee, 2018) developed lung segmentation method for automatic JPN detection in the lung CT image. Chan-Vase model was used to extract the lung contour. On the resultant images, Bayesian approach was applied to predict the exact lung contour for inclusion of JPN. Among the detected JPNs, false positive JPN were eliminated by concave points detection and circle/ellipse Hough transformation. Model was evaluated on 16,873 CT images of 84 patients, among that 42 patients 314 images with JPNs from Wonkwong University Hospital (WKUH). Method achieved JPN detection rate of $96 \%$.

(X Xiao, J Zhao, Y Qiang, H Wang, Y Xiao, X Zhang., \& Y Zhang, 2018) presented automated framework for JPNs segmentation from lung CT images. In this proposed method iterative thresholding algorithm was used to on sequence of CT slices to extract lung parenchyma. Fractal geometry method was applied on segmented lung parenchyma images to detect concave areas in lung boundary (exclusion of JPNs results in concavity in lung boundary). Successful inclusion of JPNs and lung boundary repair was achieved with convex hull algorithm. Proposed framework achieved pixel accuracy of 92.45 and Intersection over Union (IoU) of 95.9\%.

(Zhang Yang, Xie Yingying, Guo Li, Zhang Zewei, Ding Weifeng, Pan Zhifang \& Qin Jing, 2019) proposed a method for segmentation of JVNs and JPNs from thoracic CT images. In this work lung parenchyma was segmented with RG and morphological operations. Kernel graph cut algorithm was used to extract the initial nodules edges. Seed points of nodules were extracted by ray casting algorithm. Finally geodesic distance based graph cut method was applied to segment JVNs and JPNs. Proposed technique achieved high accuracy in JVNs and JPNs segmentation.

\section{PROPOSED METHODOLOGY}

This research paper presents the research work for the accurate segmentation of JPN nodules. Iterative thresholding algorithm and proposed Automated Seed Selection Region Growing algorithm (ASSRGA) are used for lung parenchyma segmentation. Centre Points Based Separation of Connected Lung Lobes Algorithm (CPBSCLLA) is proposed key algorithm to separate the connected lung lobes. Boundary pixels and concave points extraction based Juxta-Pleural Nodule segmentation algorithm is proposed for the segmentation and separation of attached pleural from the JPN.

As lung nodules are associated with their own set of properties, detection procedures for different types of lung nodules are different. Well circumscribed, JVN, JPN and GGO nodules demands specific type of segmentation method as nodules differs very much in their properties. Thus in medical field the main concern is always to detect different types of nodules at their earlier stage with high accuracy. Main difficulty in JPN segmentation is separation of pleural attached to the nodule as both have similar intensity values and non-spherical shape of nodules. As discussed in literature CAD systems from many researchers have been developed, but accurate segmentation of different types of pulmonary nodules remains one of challenging research area. Poor contrast and indistinct boundary from surrounding tissues of non-solid and part solid nodules makes their detection very difficult and undetected nodules get converts into malignant tumors. High intensity inhomogeneity and boundary 
Figure 4. Flow diagram of proposed method for segmentation of Juxta-Pleural Nodules
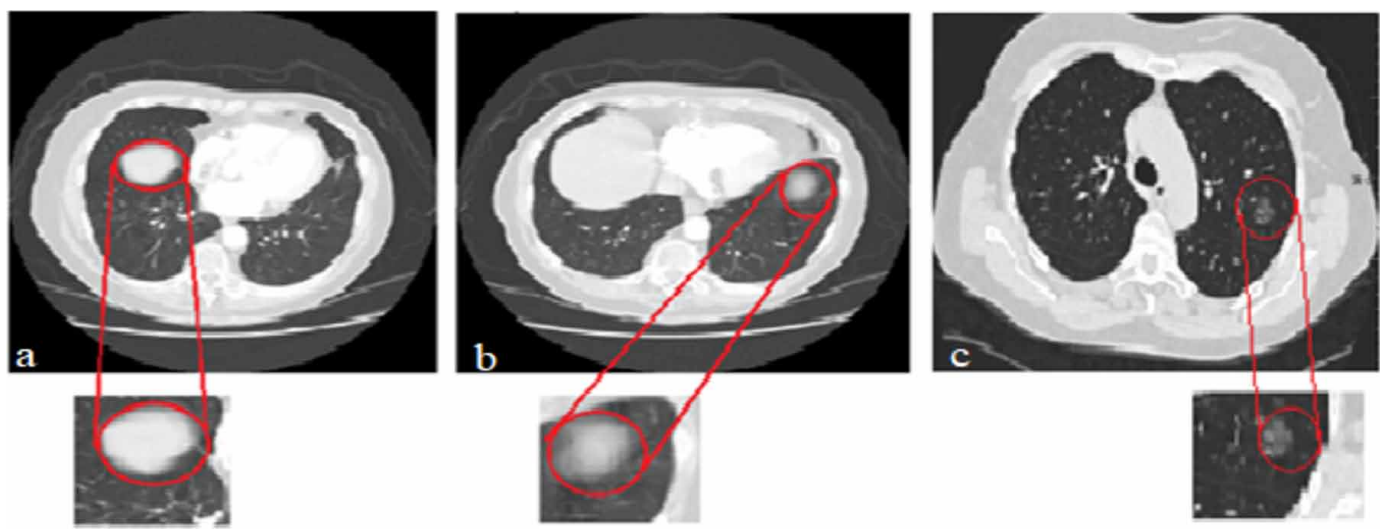

irregularity of part solid nodules also challenging parameters for segmentation. Tradition methods such intensity based method, Region Growing method and other fails to detect the JPN correctly and sometime causes under segmentation.

\section{Lung Parenchyma Segmentation}

Apply iterative thresholding algorithm to obtain binary output image.

Algorithm-1: Iterative Thresholding Algorithm

Input: CT lung image

Output: Threshold image

Step 1: Let $\mathrm{T}_{\mathrm{h}}$ is highest grey level in input image $\mathrm{Tm}$ is lowest gray level in input image.

$\mathrm{T}_{\mathrm{h}}$ and $\mathrm{T}_{\mathrm{m}}$ are obtained from histogram of input image.

$T=\left(T_{h}+T_{m}\right) / 2$

Step 2: Segment the image using $\mathrm{T}$

$T_{i}=\left\{\begin{array}{l}0, C T(x, y)>T \ldots \text { Foreground }\left(F_{g}\right) \\ 1, C T(x, y)<T \ldots \text { Background }\left(B_{g}\right)\end{array}\right.$

Step 3: Using $\mathrm{F}_{g}$ and $\mathrm{B}_{\mathrm{g}}$ calculate $\mathrm{Ti}$

$T_{i}=\left(F_{g}+B_{g}\right) / 2$

Step 4: Repeat steps 2 and 3 until $T_{i}-T_{i+1}$ is small enough that further thresholding

generates output with no further changes.

Output image of iterative thresholding contains the artefacts such as thorax, CT examination bed and others small parts. These artefacts results in increase in nodule search space. Thus the artefacts present in output binary image of algorithm-1 are eliminated by Automated Seed Selection Region 
Growing algorithm as given in algorithm-2. Algorithm automatically calculates seed points required for region grow. Thus it is fully automatic with no user interaction. Artefacts cleared binary output image contains only lung lobes.

Algorithm-2: Automated Seed Selection Region Growing Algorithm

Input: Output of Threshold image of Algorithm-1

Output: Segmented image with only lung lobes

Step 1:

a. Sequences of black colour pixels are identified by scanning binary input image in

horizontal direction starting with leftmost

pixel to right most pixel.

b. In vertical direction starting top most pixel to bottom most pixel.

c. First black pixel in the set is considered as seed

point for automatic region growing

Step 2: Let UA is set of is all unallocated pixels and $R_{i}$ possible seed regions,

$U A=\left\{x \notin \bigcup_{i=1}^{n} R_{i}: P(x) \cap \bigcup_{i=1}^{n} A_{i} \neq \varphi\right\}$

where $i=(1,2, \ldots \ldots . . n)$ and $P(x)$ is

either 4 - connected or 8 - connected

$$
\text { neighbours of pixel } x \text {. }
$$

Step 3: If $\mathrm{P}(\mathrm{x})$ and $\mathrm{R}_{j}$ intersect, then calculate $\delta(x)$, a similarity or difference measure between

pixel $\mathrm{x}$ and intersection region $\mathrm{R}_{\mathrm{j}}$. Where $\delta(x)$ is

defined as

$\delta(x)=\left|g_{v}(x)-\operatorname{mean}\left\{g_{v}(z)\right\}\right|$

where, gray level at pixel $x$ is $g_{v}(x)$ and mean gray

levels of all pixels $z$ in the region $R_{j}$ is

$$
g_{v}(z) \text { and } j=(1,2,3 \ldots . . . n) \text {. Equation (5) is }
$$

calculated for all the intersected regions $R_{j}$

with $\mathrm{P}(\mathrm{x})$ and minimum $\delta(x)$ is considered for

inclusion of pixel $x$ to that region.

Step 4: Repeat Step 3 for all pixel $x \in U A$, add a pixel y that satisfies the equation (4).

Figure 5a shows the output binary image of Algorithm-1 and Algorithm-2 outputs are shown in Figure 5c and 5b.

In some patient's cases, CT generates the images with connected lobes at upper part. To achieve the higher accuracy of JPN detection, connected lung lobes separation takes highest importance. In this work Centre Points Based Separation of Connected Lung Lobes is proposed to separate the connected lung lobes.

Algorithm-3 Centre Points Based Separation of Connected Lung Lobes Algorithm 
Figure 5. a) Output of Iterative thresholding algorithm b) Output of ASSRG algorithm without artefacts and removed small objects (c) Extracted lung ROI

Figure 3

File Edit View Insert Tools Desktop Window Help

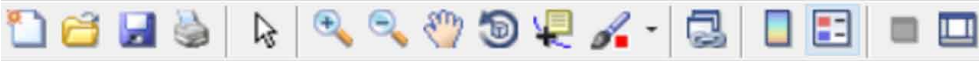

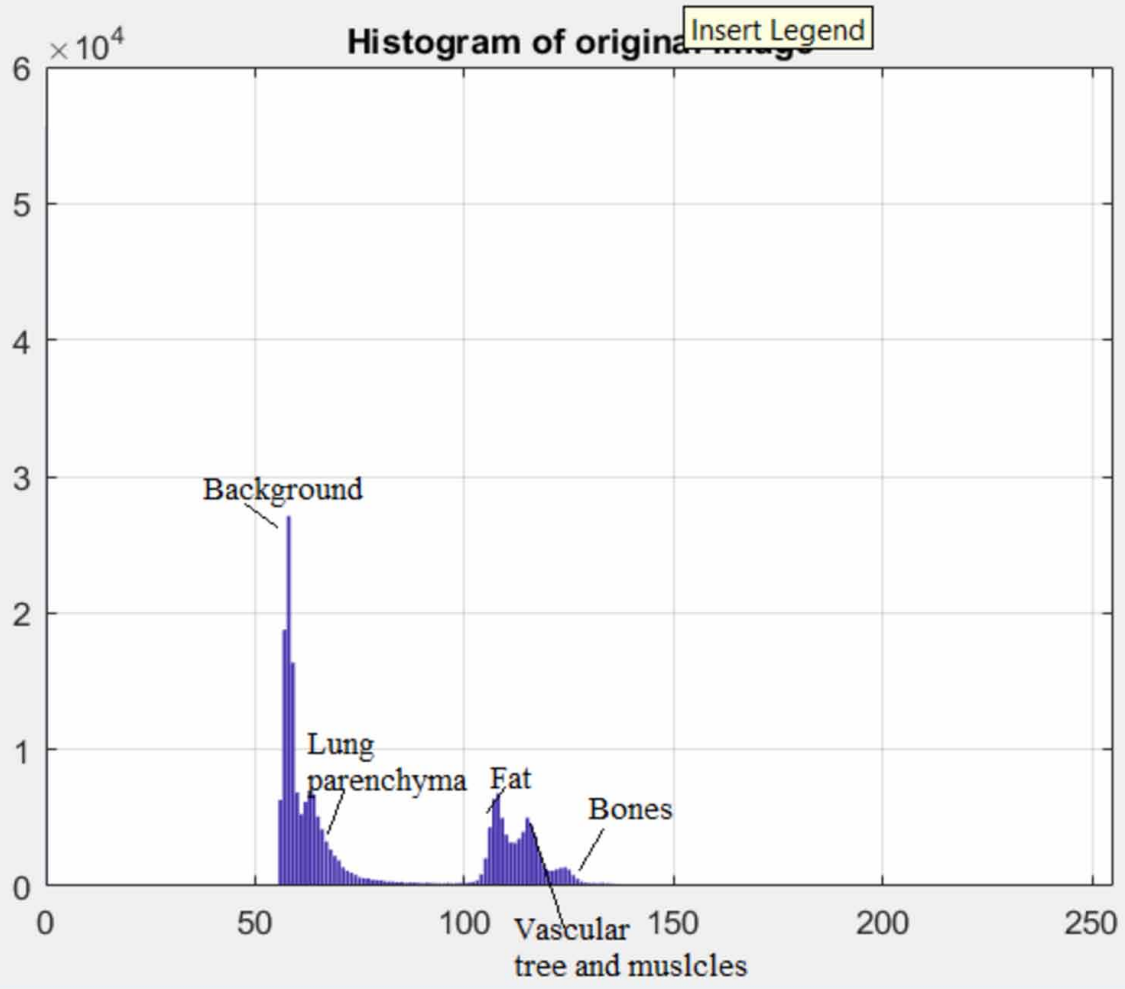

Input: Output of Algorithm - 2

Output: Segmented image with separated left and right lung lobes Step 1: Apply 3D connecting component algorithm

Ignore small components.

if (one large connected component)

Lobes are connected go to step 2.

else

$$
\text { Lobes are not connected and exit. }
$$

Step 2: Let $l u(x 1, y 1), r u(x 2, y 2), \quad l l(x 3, y 3), r l(x 4, y 4)$ are left upper corner point, right upper corner point, left lower corner point and right lower corner point respectively.

a. Using above four corner points determine left centre point cl(x5, y5), right centre

point $\operatorname{cr}\left(\mathrm{x}_{6}, \mathrm{y}^{6}\right)$, upper centre point uc $\left(\mathrm{x}^{7}, \mathrm{y}^{7}\right)$ and lower centre point lc $(\mathrm{x} 8, \mathrm{y} 8)$.

b. Using $\mathrm{cl}\left(\mathrm{x} 5, \mathrm{y}^{5}\right), \mathrm{cr}\left(\mathrm{x}_{6}, \mathrm{y}^{6}\right), \mathrm{uc}\left(\mathrm{x} 7, \mathrm{y}^{7}\right)$ and $\mathrm{lc}_{\mathrm{c}}(\mathrm{x} 8$, $\mathrm{y} 8)$, lung parenchyma centre point $\mathrm{P}(\mathrm{x}, \mathrm{y})$ is determined. 
Figure 6. a) Output of ASSRG algorithm with connected lung lobes b) Considered points on image c) Left and Right lobes separated
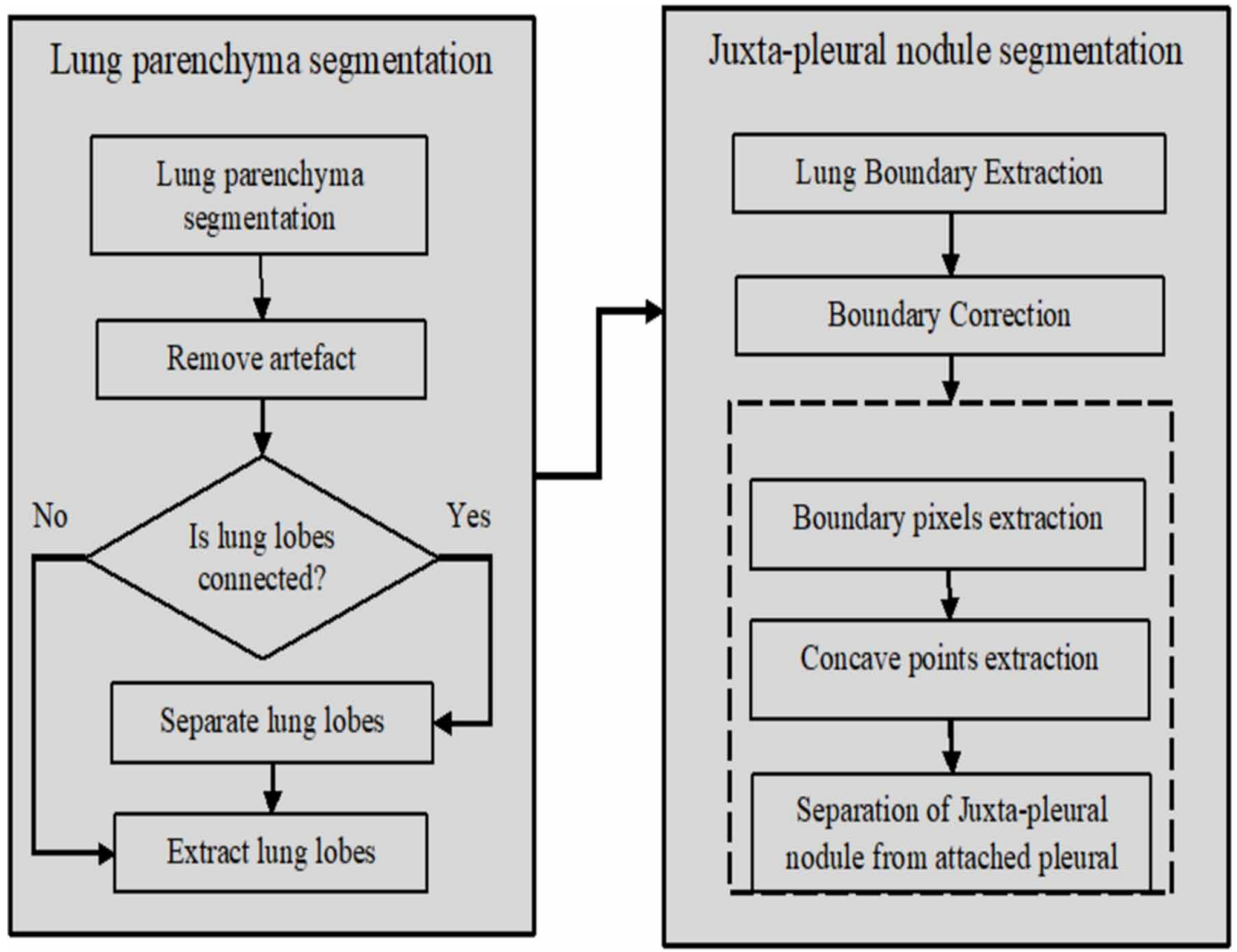

c. Starting with $\mathrm{P}(\mathrm{x}, \mathrm{y})$ scan the line upward direction, until the point $L(x c, y c)$ with lower intensity is identified. $\mathrm{L}(\mathrm{xc}, \mathrm{yc})$ is centre of connected region.

d. Using $\mathrm{L}(\mathrm{xc}, \mathrm{yc})$ determine extreme points of connected region $\mathrm{L}(\mathrm{xu}, \mathrm{yu})$ and $\mathrm{L}(\mathrm{xl}, \mathrm{yl})$.

e. Apply morphology erosion from two identified extreme points to separate the connected lung lobes.

Step 3: Using left centre point on image boundary cl (x5, y5) as starting point traverse along the

straight line toward right, until point a with three consecutive $1^{\prime} \mathrm{s}$ are identified.

step 4: Using pixel al as starting point and its 8-neighborhood pixels obtain boundary pixels of

left lung. Let set $A\left\{a_{1}, a_{2}, a_{3} \ldots . . . a_{n}\right\}$ represents left lung boundary.

Step 5: Repeat the step 1 using cr $(x 6, y 6)$ as starting point and traverse along straight line towards left, until point bl with three consecutive $1^{\prime}$ s are identified.

Step 6: Using pixel bl as starting point and its 8-neighborhood pixels obtain boundary pixels of 
Figure 7. a) Original CT image with Juxta-Pleural Nodule b) segmented lung lobes c) Lung boundary with exclusion of JuxtaPleural Nodule
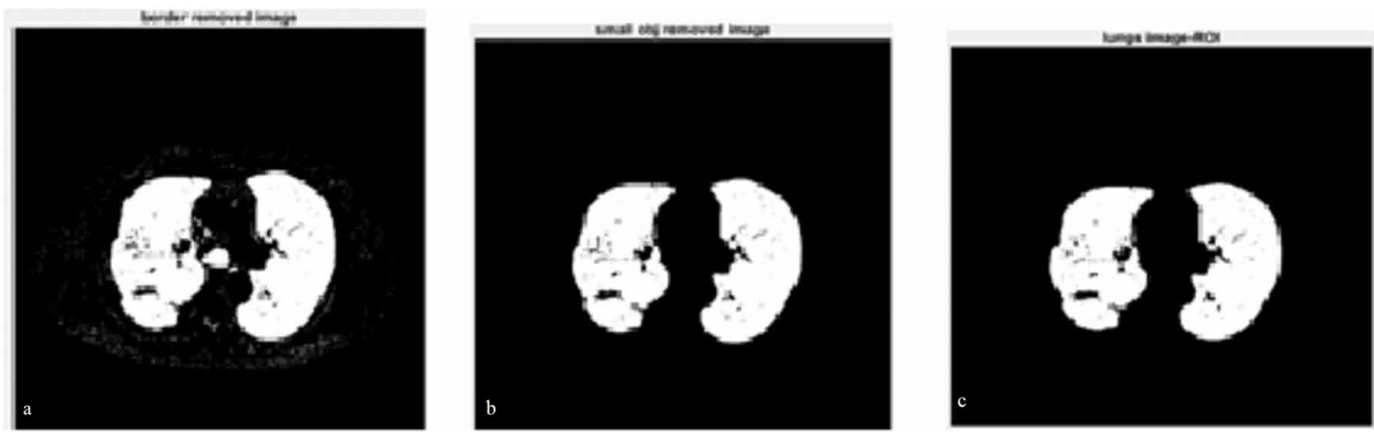

right lung. Let set $A\left\{b_{1}, b_{2}, b_{3} \ldots . . . b_{n}\right\}$ represents

right lung boundary .

Figure 6a shows the connected lung lobes CT image, Figure $6 \mathrm{~b}$ shows the selected points on image and $6 \mathrm{c}$ shows the output of the algorithm-3.

\section{Juxta-Pleural Nodule segmentation}

As the intensity values of both attached pleural and JPN are same, traditional parenchyma segmentation procedures results in concavity by exclusion of present JPN. The key features of Juxta-pleural nodule segmentation algorithm are extraction of boundary pixels, extraction of concave points and separation of JPN from attached pleural.

Figure 7a is CT image with JPN, segmented lung lobes with excluded JNP are as shown in Figure $7 \mathrm{~b}$ and $7 \mathrm{c}$. Exclusion of JPNs results in under segmentation and entire lung parenchyma segmentation becomes inefficient. Thus to include and segment the JPNs accurately we proposed concavity boundary pixels extraction method that uses lung boundary pixels information to segment JPNs.

Algorithm-4 Juxta-Pleural Nodule Segmentation

Boundary Pixels Extraction

The extracted lung boundary is very rough with sharp edges. So before extraction of JPNs lung boundary is smoothed by the lung boundary smoothing algorithm by (S. Hu, K. Bi, Q. Ge, M. Li, X. xie \& $\mathrm{X}$. Xiang, 2014).

Let smoothened boundary of left lung is Left boundary with M points such that,

Left $t_{\text {boundary }}=\left\{L\left(m_{i}\left(x_{i}, y_{i}\right) ; i=1 \ldots . . M\right\}\right.$, where $M$ is number of left lung boundary points. $m_{i}$ is $i^{\text {th }}$ left lung boundary point with $m_{i+1}$ and $m_{i-1}$ immediate neighbor points.

Similarly boundary points set of right lung is Right boundary $_{\text {such }}$ that,

$\operatorname{Right}_{\text {boundary }}=\left\{R\left(n_{i}\left(x_{i}, y_{i}\right) ; i=1 \ldots . . N\right\}\right.$, where $N$ is number of right lung boundary points. $n_{i}$ is $i^{\text {th }}$ right lung boundary point with $\mathrm{n}_{\mathrm{i}+1}$ and $\mathrm{n}_{\mathrm{i}-1}$ immediate neighbor points. Using the boundary points concave points are determined.

\section{Concave Points Extraction}

In this proposed method the procedure to determine the concave points consist of 
a. Determine the corner points using Ramer-Douglas-Peucker (RDP) algorithm.

b. Using the corner points, extract concave points using proposed algorithm Concave Points of Juxta Pleural Nodules (CPJPN)

Left $_{\text {boundary }}$ and Right ${ }_{\text {boundary }}$ are inputs for RPD algorithm. For these inputs algorithms returns all possible corner present in both Left ${ }_{\text {boundary }}$ and Right ${ }_{\text {boundary }}$. Let the sets Left $t_{\text {corner }}=\left\{1_{\text {corner, } i}\left(\mathrm{x}_{\mathrm{i}}, \mathrm{y}_{\mathrm{i}}\right)\right.$; $1_{\text {corner }, \mathrm{i}} \in$ Left $\left._{\text {boundary }}\right\}$ and Right $\mathrm{corner}=\operatorname{Left}_{\text {corner }}=\left\{\mathrm{r}_{\text {corner }, \mathrm{i}}\left(\mathrm{x}_{\mathrm{i}}, \mathrm{y}_{\mathrm{i}}\right) ; \mathrm{r}_{\text {corner }, \mathrm{i}} \in \mathrm{Right}_{\text {boundary }}\right\}$ represents presence of possible corner points in left and right lungs boundary.

Using the corner points Left corner $_{\text {and Right }}$ corner sets, concave points are computed using proposed CPJPN that represents possibility of presence of JPNs. Algorithm proceeds in clockwise direction by connecting possible points until second corner point is connected. The vectors $\mathrm{V}_{i}$ contains 1 $1_{\text {corner,i }}$ and vector $\mathrm{V}_{\mathrm{i}+1}$ contains $1_{\text {corner,i, }} 1_{\text {corner, },+1 \text {. }}$ The required orientation for considered corner points is calculated as cross product of $\mathrm{V}_{\mathrm{i}}$ and $\mathrm{V}_{\mathrm{i}+1}$. Finally left and right lungs boundary orientation is computed. Any breaks in traversal gives the concave points.

Algorithm-4a Concave Points of Juxta-Pleural Nodules

Input: Output of Algorithm - 3

Output: Extracted concave points

Step 1: For Leftboundary obtain $l_{\text {corner,i-1, }} l_{\text {corner, } i}$, and $l_{\text {conner,i+1 }}$ points

Step 2: For $1_{\text {corner }} i=1$, Repeat the steps 2,3,4,5 until $i=M$ if $i==1$

$l_{\text {corner, } i-1}=l_{\text {corner, }{ }^{\prime}}$, where $M$ is last corner point. Same in case of $r_{\text {corner }}$ set

else if $i==M$

$1_{\text {corner,i+1 }} 1_{\text {corner, } 1}$

Step 3: Calculate vectors $V_{i}$ and $V_{i+1}$

$V_{i}=\left(l_{\text {corner, } i-1,} l_{\text {corner, } i}\right)$ and $V_{i+1}=\left(l_{\text {corner, } i}, l_{\text {corner, } i+1}\right)$

Step 4: Orin $[i]=\operatorname{SIGN}\left(\mathrm{V}_{i} \star \mathrm{V}_{i+1}\right)$

Step 5: SumOrin = SumOrin + Orin[i]

Step 6: Sumorin = SIGN (SumOrin)

Step 7: For $i=1$ Repeat until $i=M$

if Orin $[i] \neq$ Sumorin

Add $l_{\text {corner, } i}$ point as concave point into $l_{\text {concave }}$

set

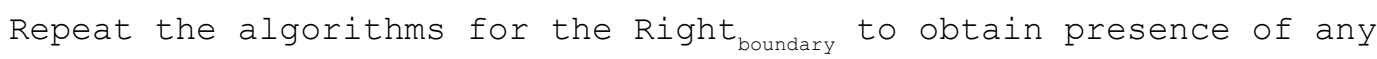

Figure 8. a) Boundary points on left and right lung b) Concave points on lung boundary
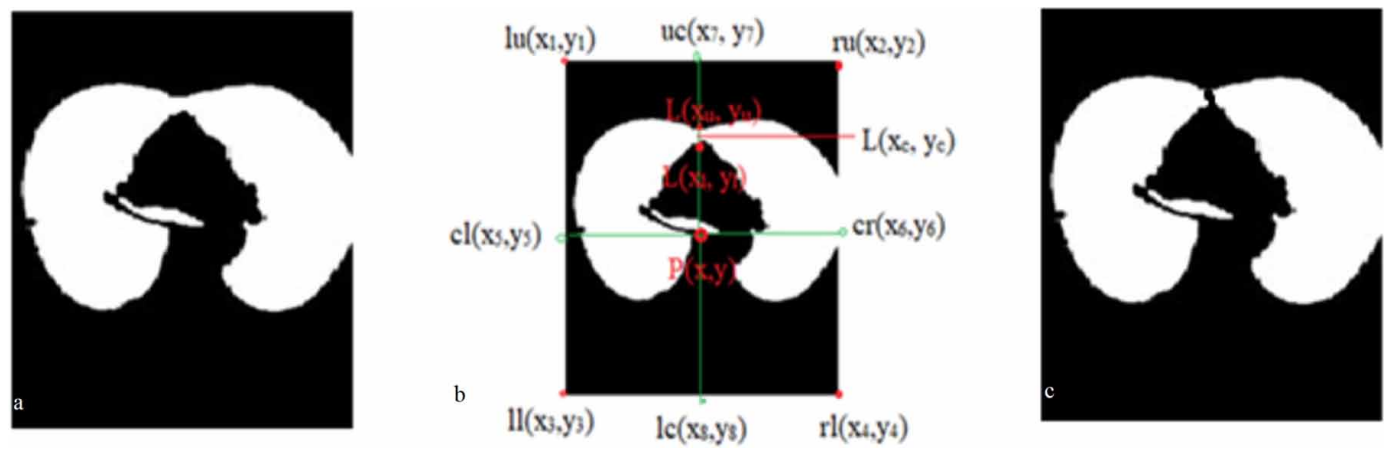
concave set $r_{\text {concave. }} l_{\text {concave }}$ and $r_{\text {concave }}$ sets represents presence of any JPNs in the left lung and right lung.

Figure 8 a shows the boundary points of JPN. The set of concave points proves the presence of JPNs and are used to segment the JPNs. Figure $8 \mathrm{~b}$ shows the concave points (red color) of JPNs.

In algorithm 4b Classical 1 Least Square Fitting is used to fit ellipse around the identified set of concaves set. Majoraxis, minoraxis and angle of orientation are used for every concave point $i$,. Aspect Ratio (AR) is calculated as ratio of majoraxis and minoraxis. This separates the attached pleural from JPN.

Algorithm-4b Separation of JPNs from Attached Pleural

Input: Output of Algorithm-4a

Output: Segmented Juxta-pleural nodule

step 1: Let $Q$ number of points in $l_{\text {concave }}$ set, for $i=1$ untill i=Q

a.

b.

Perform elliptical fitting to segment $l_{\text {concave }}$

Calculate the Aspect Ratio (AR) as

$$
A=\frac{\text { majoraxis }}{\min \text { oraxis }}
$$

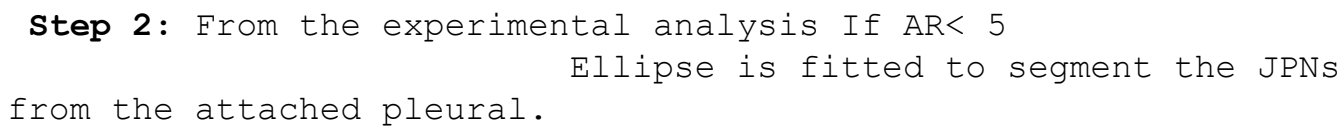

\section{DATA SET}

Lung cancer is major issue in health care community. In a view to encourage lung cancer research, public database Lung Image Database Consortium-Image Database Resource Initiative (LIDC-IDRI) was founded in 2004 and currently database has CT images of 1080 patients (cancerimagingarchive. net; Michael Samir Labib habib, 2009). The database also provides the Ground Truth associated with each nodules to promote the research on lung cancer. From this database, 85 cases CT lung images are considered for evaluation of proposed methodology.

\section{RESULTS AND DISCUSSION}

Lung parenchyma segmentation results for same CT lung images without and with JPN is as shown in Figure 9 for proposed method and other considered methods such as Watershed and Region growing methods. The images in Figure 9 are given as: Row 1 describes (a) original CT lung image without JPN, (b) segmentation result of Watershed method (c) segmentation result of Region growing method and (d) segmentation result of proposed method. From Figure 9(b), (c) and (d), it is concluded that all the three methods provides same segmentation result for CT image without JPN. Row2 describes (e) original CT lung image with JPN, (f) and (g) failure of Watershed and Region growing method for inclusion of JPN, (h) successful inclusion of JPN by proposed method and Row3 (i) and (j) shows the segmentation of JPN by proposed method.

To evaluate the results of proposed and other two considered methods, two quantitative metrics: time complexity and Pixel Accuracy, two contour based metrics: Mean Distance and Hausdorff Distance and based on confusion metric Accuracy, sensitivity, specificity and overlap were computed.

\section{Pixel Accuracy (Pacc)}

Pixel accuracy is percentage of pixels identified correctly. Higher pixel accuracy results in accurate segmentation. The Pacc is calculated as, 
Figure 9. row1 a to d segmentation result of all three methods for CT lung image without JPN, row2 e. CT image with JPN. $f$ and $g$ exclusion of JPN by watershed and region growing methods. row2 and row $3 \mathrm{~h}$ to $\mathrm{j}$ segmented JPN by proposed method.
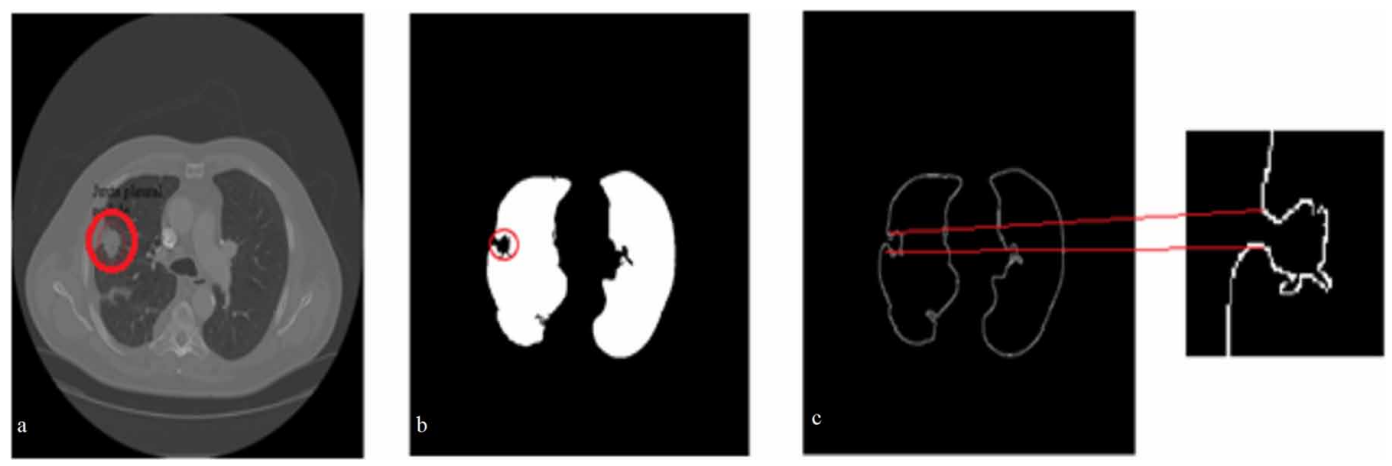

$$
P a c c=\frac{\sum_{i=0} p_{i 1}}{\sum_{i=0} \sum_{j=0} g_{j 1}}
$$

Where,

$\mathrm{S}_{\mathrm{i}}$ - Correctly identified pixels, $\mathrm{S}_{\mathrm{i}}=\left\{\mathrm{s}_{\mathrm{i} 1} \mathrm{~s}_{\mathrm{i} 2}, \ldots \ldots \ldots \mathrm{s}_{\mathrm{im}}\right\}$

$\mathrm{G}_{\mathrm{j}}$-Total number of pixels, $\mathrm{G}_{\mathrm{j}}=\left\{\mathrm{g}_{\mathrm{j} 1}, \mathrm{~g}_{\mathrm{j} 2}, \ldots \ldots \ldots . \mathrm{g}_{\mathrm{jm}}\right\}$

\section{Mean Distance (MD)}

Let A is segmented nodule contour and B is Ground Truth contour. XOR operation on A and B gives region $\mathrm{C}$. Apply $\mathrm{K}^{\text {th }}$ normal on auxiliary boundary of $\mathrm{C}$ to obtain contours intersects points (x1, y1) and $(\mathrm{x} 2, \mathrm{y} 2)$. Euclidian distance of $(\mathrm{x} 1, \mathrm{y} 1)$ and $(\mathrm{x} 2, \mathrm{y} 2)$ is $\mathrm{D}_{\mathrm{k}}$. MD calculated as,

$M D=\frac{1}{N} \sum_{K=1}^{N} D_{k}$

Where,

$\mathrm{N}$ - total number of boundary pixels.

\section{Hausdorff Distance (HD)}

The difference of maximum distance between the two contours A and B is Hausdorff Distance

$$
H D(A, B)=\max \left(h_{m}(A, B), h_{m}(B, B)\right)
$$

Where, 
$h_{m}=\max _{q \in A}(D \min (q, B)$,

$h_{m}$ is maximum of minimum distance of points $\mathrm{q}$ of contour A to contour B

\section{Four Region Based Metrics}

Overlap, accuracy, sensitivity and specificity are computed as below

Let

Total count of True Positive pixels - TP,

Total count of True Negative pixels - TN,

Total count of False Positive pixels - FP

Total count of False Negative pixels - FN,

Overlap $=\frac{T P}{T P+F P+F N}$

Accuracy $=\frac{T P+T N}{T P+T N+F P+F N}$

Sensitivity $=\frac{T P}{T P+F N}$

Specificity $=\frac{T N}{T N+F P}$

The minimum time, average time and maximum time taken by three methods are shown in Figure 10a. From the Figure 10b, Pixel Accuracy of proposed method is better than other two methods. Figure 11 is Box plots for proposed and other two methods and shows that performance of proposed method is outstanding compared to other two methods.

\section{CONCLUSION}

Early detection of Juxta-Pleural Nodules significantly increases life span of patients suffering with lung cancer due to Juxta-Pleural Nodules. CAD systems based on traditional segmentation techniques such as thresholding, RG, FCM and other implemented by many researchers had failed for segmentation of Juxta-Pleural Nodules with a result of under segmentation. In this study, automated segmentation of Juxta Pleural Nodule method was proposed. In lung parenchyma segmentation step iterative thresholoding, two newly designed automated seed selection region growing algorithm and center points based separation of connected lung lobes algorithm were applied to extract lung 
Figure 10. a) Time complexity of all three methods b) Comparison of pixel accuracy of all three methods
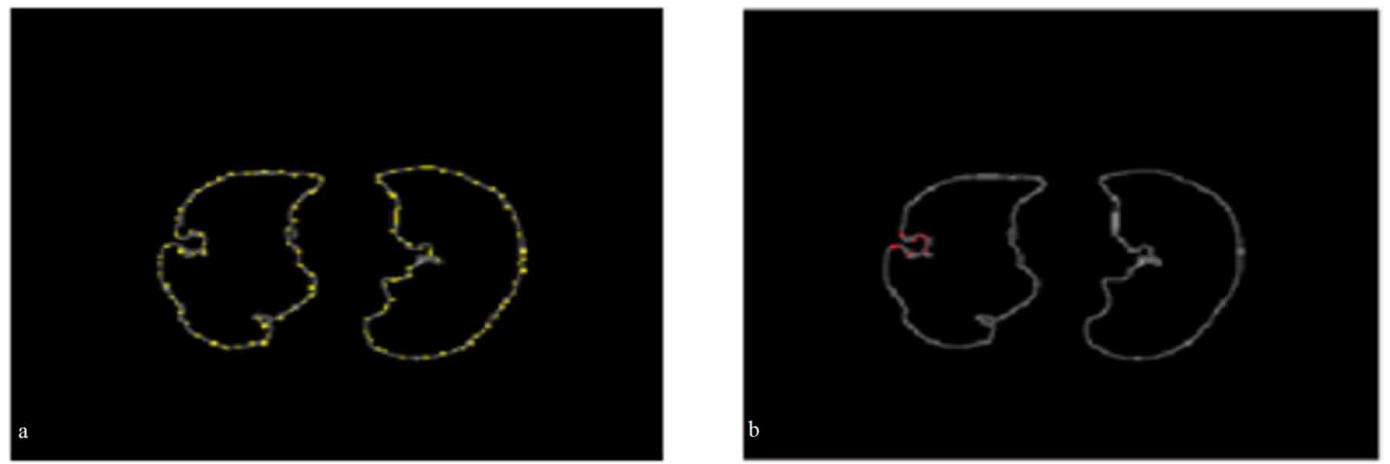

Figure 11. Box plot of all considered metrics for three methods a) Mean distance b) Hausdorff Distance c) accuracy d) sensitivity e) specificity and d) overlap
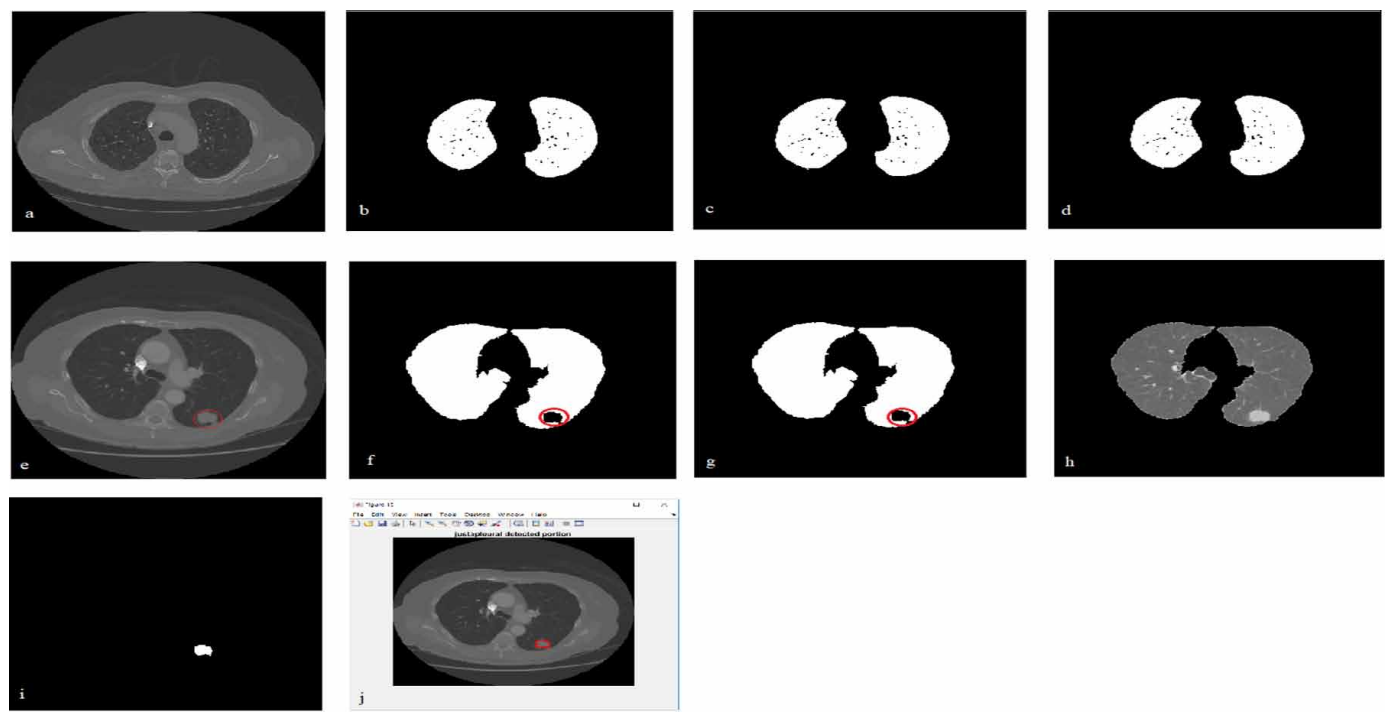

lobes and to separate connected lung lobes respectively. Boundary pixels extraction and identified concave pixels extraction based, novel Juxta-Pleural nodules segmentation algorithm on CT image was proposed to segment the Juxta-Pleural nodules. 85 cases CT images from LIDC public database were considered to analyze segmentation performance of our method. We choose LIDC as it contains the information about the nodules such as its type, location, size, disease stage and other information. Segmentation result comparison of proposed method, watershed and RG methods was considered in this study. Detailed result analysis showed that time complexity of $1.12 \mathrm{sec}$, Pixel accuracy of on average $94 \%$, Acuracy of $98 \%$, specificity of $97 \%$, better sensitivity and overlap were achieved with proposed method. As compared with watershed and RG methods, our system obtained higher Juxtapleural Nodules segmentation accuracy.

As Juxta-Pleural Nodules segmentation accuracy of our method is high, the main practical significances of our method are, it can be used as core module in lung cancer detection CAD system to segment early stage Juxta-Pleural in less time, with help of this model radiologist can get valuable information about Juxta-Pleural Nodules and thus draw a useful conclusion on treatment plan. In this work, segmentation framework for only Juxta-Pleural nodules segmentation is proposed. In future work, proposed algorithm will be explored to segment the early stage JVN, and GGO nodules. 


\section{REFERENCES}

Acharjya, D. P., \& Chowdhary, C. L. (2018). Breast Cancer Detection Using Hybrid Computational Intelligence Techniques. In Handbook of Research on Emerging Perspectives on Healthcare Information Systems and Informatics. IGI Global.

Campos, D. M., Simões, A., Ramos, I., \& Campilho, A. (2014). Feature-Based Supervised Lung Nodule Segmentation. 1st International Conference on Health Informatics, 42, 23-26. doi:10.1007/978-3-319-03005-0_7

Chowdhary, C. L., \& Acharjya, D. (2018). Segmentation of Mammograms Using a Novel Intuitionistic Possibilistic Fuzzy C-Mean Clustering Algorithm. Nature Inspired Computing, 75-82.

Chowdhary, C. L., \& Acharjya, D. P. (2016). A hybrid scheme for breast cancer detection using intuitionistic fuzzy rough set technique. International Journal of Healthcare Information Systems and Informatics, 11(2), 38-61. doi:10.4018/IJHISI.2016040103

Chowdhary, C. L., \& Acharjya, D. P. (2016). Breast cancer detection using intuitionistic fuzzy histogram hyperbolization and possibilistic fuzzy C-mean clustering algorithms with texture feature based classification on mammography images. AMC International Conference Proceedings Series, 12.

Chowdhary, C. L., \& Acharjya, D. P. (2017). Clustering algorithm in possibilistic exponential fuzzy c-mean segmenting medical images. Journal of Biomimetics. Biomaterials and Biomedical Engineering, 30, 12-23. doi:10.4028/www.scientific.net/JBBBE.30.12

Chowdhary, C. L., Sai, G. V. K., \& Acharjya, D. P. (2016). Decrease in false assumption for detection using digital mammography. Computational Intelligence in Data Mining, Springer, 2, 325-333. doi:10.1007/978-81322-2731-1_30

Chung, Ko, Jeon, Yoon, \& Lee. (2018). Automatic Lung Segmentation with Juxta-Pleural Nodule Identification Using Active Contour Model and Bayesian Approach. IEEE J Trans. Eng Health Med., 6.

Diciotti, S., Picozzi, G., Falchini, M., Mascalchi, M., Villari, N., \& Valli, G. (2008). 3-D segmentation algorithm of small lung nodules in spiral CT images. IEEE Transactions on Information Technology in Biomedicine, 12(1), 7-19. doi:10.1109/TITB.2007.899504

Ezhil, E., \& Nithila, S. S. (2016). Segmentation of lung nodule in CT data using active contour model and Fuzzy C-mean clustering. Alexandria Engineering Journal, 55(3), 2583-2588. doi:10.1016/j.aej.2016.06.002

Farag, H. E. A., El Munim, J. H., Graham, J. H., \& Farag, A. A. (2013). A novel approach for lung nodules segmentation in chest CT using level sets. IEEE Transactions on Image Processing, 22(12), 5202-5213. doi:10.1109/TIP.2013.2282899

Geng, H., Bian, Z., Yang, J., Tan, W., \& Zhao, D. (2014). Fully automatic extraction of lung parenchyma from CT scans. Proceedings of the 2014 11th World Congress on Intelligent Control and Automation (WCICA), 5626-5630. doi:10.1109/WCICA.2014.7053678

Gong, J., Wang, L., \& Wang, Y. (2015). Segmentation of lung parenchyma based on gray-level integrated projection and fuzzy C-Means clustering algorithm. Zhongguo Shengwu Yixue Gongcheng Xuebao, 34, 109-113.

Hu, S., Bi, K., Ge, Q., Li, M., Xie, X., \& Xiang, X. (2014). Curvature-based correction algorithm for automatic lung segmentation on chest CT images. Bio Systems, 22(01), 1-28. doi:10.1142/S0218339014500016

Kubota, T., Jerebko, A. K., Dewan, M., Salganicoff, M., \& Krishnan, A. (2011). Segmentation of pulmonary nodules of various densities with morphological approaches and convexity models. Medical Image Analysis, 15(1), 133-154. doi:10.1016/j.media.2010.08.005

Li, J., Zheng, B., Liang, H., \& Deng, Y. L. (2013). Segmentation research of pulmonary parenchyma based on improved convex hull algorithm. Zhongguo Shengwu Yixue Gongcheng Xuebao, 32, 484-490.

Mansoor, U., Bagci, Z., Xu, B., Foster, K. N., Olivier, K. N., Elinoff, J. M., Suffredini, A. F., Udupa, J. K., \& Mollura, D. J. (2014). A generic approach to pathological lung segmentation. IEEE Transactions on Medical Imaging, 33(12), 2293-2310. doi:10.1109/TMI.2014.2337057 
Messay, T., Hardie, R. C., \& Rogers, S. K. (2010). A new computationally efficient CAD system for pulmonary nodule detection in CT imagery. Medical Image Analysis, 14(3), 390-406. doi:10.1016/j.media.2010.02.004

Qi, C., \& Yu, W. (2010). Research on Ground Moving Target Indication for Spaceborne MIMO-SAR of Multiple Carrier-frequencies. J. Electron. Inf. Technol., 32(10), 1100-1104. doi:10.3724/SP.J.1146.2009.01343

Samir \& Habib. (2009). A Computer Aided Diagnosis System (CAD) for the Detection of Pulmonary Nodules on CT Scans. Systems and Biomedical Engineering Department, Faculty of Engineering, Cairo University, Giza, Egypt.

Sharma, A., Ansari, M. D., \& Kumar, R. (2018). A comparative study of edge detectors in digital image processing. $4^{\text {th }}$ International Conference on Signal Processing, Computing and Control (ISPCC), IEEE Trans. doi:10.1109/ISPCC.2017.8269683

Sharma \& Kumar. (2019). A Constrained Framework for Context-Aware Remote E-healthcare (CARE) Services. Trans. on Emerging Telecommunication Technology. 10.1002/ett.3649

Suzuki, K., \& Doi, K. (2004). Characteristics of a Massive Training Artificial Neural Network in the distinction between lung nodules and vessels in CT images. Proc. CARS 1268, 923-928. doi:10.1016/j.ics.2004.03.037

Suzuki, K., \& Doi, K. (2005). How can a massive training artificial neural network (MTANN) be trained with a small number of cases in the distinction between nodules and vessels in thoracic CT? Academic Radiology, 12(10), 1333-1341. doi:10.1016/j.acra.2005.06.017

Suzuki, K., Shi, Z. H., \& Zhang, J. (2008). Supervised enhancement of lung nodules by use of a Massive-Training Artificial Neural Network (MTANN) in Computer-Aided Diagnosis (CAD). Proc. ICPR, 680-683. doi:10.1109/ ICPR.2008.4761114

Tan, Schwartz \& Zhao. (2013). Segmentation of lung lesions on CT scans using watershed, active contours, and Markov random field. Medical Physics. International Journal of Medical Physics Research and Practice, 40(4). 10.1118/1.4793409

The Cancer Imaging Archive. (2019). Frederick Nat. Lab for Cancer Research. https://wiki.cancerimagingarchive. net/display/Public/LIDC-IDRI

Tong, J., Lu, M., Zhao, D. Z., \& Wang, X. (2008). Automatic Lung Parenchyma Segmentation on CT Image. Journal of Northeastern University, 29(7), 965-975.

Wang, Q., Song, E., Jin, R., Han, P., Wang, X., Zhou, Y., \& Zeng, J. (2009). Segmentation of lung nodules in computed tomography images using dynamic programming and multidirection fusion techniques. Academic Radiology, 16(6), 678-688. doi:10.1016/j.acra.2008.12.019

Xiao, X., Zhao, J., Qiang, Y., Wang, H., Xiao, Y., Zhang, X., \& Zhang, Y. (2018). An Automated Segmentation Method for Lung Parenchyma Image Sequences Based on Fractal Geometry and Convex Hull Algorithm. Applied Sciences (Basel, Switzerland), 8(5), 1-15. doi:10.3390/app8050832

Xuanping, Wang, Dai, \& Zhang. (2015). Supervised recursive segmentation of volumetric CT images for 3D reconstruction of lung and vessel tree. Computer Methods and Programs in Biomedicine, 122, 316-329.

Yang, Z., Xie, Y., Li, G., Zhang, Z., Ding, W., Pan, Z., \& Jing, Q. (2019). Robust Pulmonary Nodule Segmentation in CT Image for Juxta-pleural and Juxta-vascular Case. Current Bioinformatics, 14(2), 139-147. doi:10.2174/ 1574893613666181029100249 
Vijayalaxmi Mekali is an Assistant Professor in Department of Computer Science and Engineering, $\mathrm{K} S$ Institute of Technology, Bangalore, affiliated to Visvesvaraya Technological University (VTU), Belgavi. She is pursuing Ph.D in VTU. Her current research interests are image processing and medical image processing.

Girijamma H. A. is working as Professor in the department of Computer science \& Engineering at RNSIT, Bangalore and having 23 years of experience in teaching. Areas of interests include automata theory, compilers, machine learning and fuzzy systems. She was awarded Ph.D in 2012. 